\title{
Assessing health disparities in breast cancer incidence burden in Tennessee: geospatial analysis
}

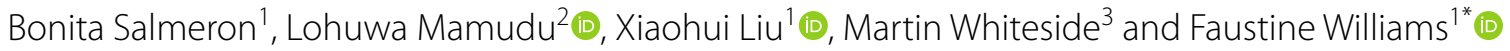

\begin{abstract}
Background: Tennessee women experience the 12th highest breast cancer mortality in the United States. We examined the geographic differences in breast cancer incidence in Tennessee between Appalachian and non-Appalachian counties from 2005 to 2015.

Methods: We used ArcGIS 10.7 geospatial analysis and logistic regression on the Tennessee Cancer Registry incidence data for adult women aged $\geq 18$ years $(N=59,287)$ who were diagnosed with breast cancer from 2005 to 2015 to evaluate distribution patterns by Appalachian county designation. The Tennessee Cancer Registry is a populationbased, central cancer registry serving the citizens of Tennessee and was established by Tennessee law to collect and monitor cancer incidence. The main outcome was breast cancer stage at diagnosis. Independent variables were age, race, marital status, type of health insurance, and county of residence.
\end{abstract}

Results: Majority of the sample were White (85.5\%), married (58.6\%), aged $\geq 70$ (31.3\%) and diagnosed with an early stage breast cancer (69.6\%). More than half of the women had public health insurance (54.2\%), followed by private health insurance coverage (44.4\%). Over half of the women resided in non-Appalachian counties, whereas $47.6 \%$ were in the Appalachian counties. We observed a significant association among breast cancer patients with respect to marital status and type of health insurance coverage $(p=<0.0001)$. While the logistic regression did not show a significant result between county of residence and breast cancer incidence, the spatial analysis revealed geographic differences between Appalachian and non-Appalachian counties. The highest incidence rates of 997.49-1164.59/100,000 were reported in 6 Appalachian counties (Anderson, Blount, Knox, Rhea, Roane, and Van Buren) compared to 3 nonAppalachian counties (Fayette, Marshall, and Williamson).

Conclusions: There is a need to expand resources in Appalachian Tennessee to enhance breast cancer screening and early detection. Using geospatial techniques can further elucidate disparities that may be overlooked in conventional linear analyses to improve women's cancer health and associated outcomes.

Keywords: Breast cancer, Health disparities, Geographic information system, Appalachian Tennessee, Nonappalachian Tennessee

*Correspondence: Faustine.Williams@nih.gov

${ }^{1}$ Division of Intramural Research, National Institute on Minority Health and Health Disparities, Bethesda, MD, USA

Full list of author information is available at the end of the article

\section{Background}

Breast cancer remains the most commonly diagnosed cancer, as well as the second leading cause of cancer death among women in the United States (U.S.). The burden of breast cancer, however, varies by the region (rural vs urban) where an individual lives $[1,2]$. The 
Appalachian region is defined as the 205,000-squaremile region that stretches from southern New York to northern Mississippi, including 420 counties over 13 states [3] (see Fig. 1). One out of every three counties in the Appalachian region is considered rural. The Appalachian geography isolates many communities from cities and healthcare resources, where the residents of this region are often described as the "invisible/neglected minority" [4]. Mountain chains, valleys, and rivers often separate small communities and inhibit transportation to healthcare services [5]. Residents of the Appalachian region experience higher rates of poverty, lower levels of literacy, and poorer health outcomes compared to the
non-Appalachian region [6, 7]. Although cancer mortality rates in the U.S. have continuously decreased since 1991, geographical and racial/ethnic disparities in cancer mortality and survival persist $[4,7,8]$ in the Appalachian region.

Figure 1 shows the map of the Appalachian states, counties, and subregions

Central Appalachian comprises 234 counties across six states. These are Eastern Kentucky, Southwest North Carolina, Southeast Ohio, Northeast Tennessee, Southwest Virginia, and West Virginia. It is the most distressed, rural, deprived, and at-risk part of Appalachia with a disproportionately high burden of cancer [9-13] and

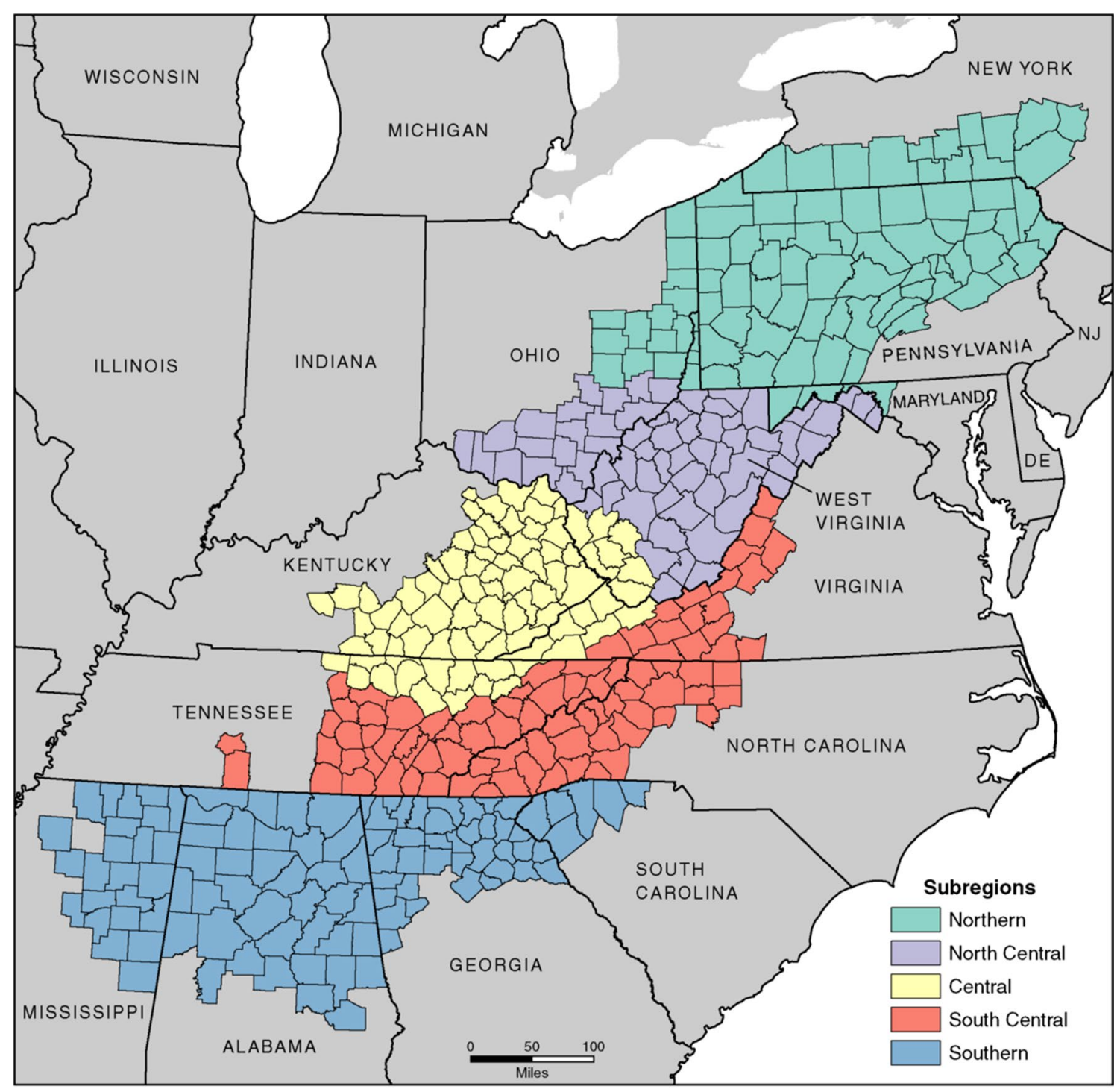

Map by: Appalachian Regional Commission, November 2009.

Fig. 1 Appalachian region. Map by Appalachian Regional Commission 
low socioeconomic characteristics [13-15], yet, existing research rarely includes Central Appalachia [3, 16, 17]. Among many challenges contributing to this significant health disparity, evidence showed that residents of rural Appalachia are at high risk for not receiving recommended cancer screening tests, including mammograms for breast cancer [10, 18-23]. Lack of cancer screening has been attributed to lack of education, unhealthy behavioral lifestyles [7, 15, 20, 24-27], of the Appalachian culture [21, 27-32], geographical variations $[10,26,33-$ $35]$ and persistent poverty $[15,26]$.

Tennessee is one of the largest and most diverse states in the Appalachian region in terms of race/ethnicity, income, and location (i.e., remote, rural, small towns, rural-urban, and urban) compared to similar states that are largely rural such as Kentucky and West Virginia. Over half (52) of the counties in Tennessee are classified as Appalachia (eastern part, mainly rural \& small towns) which tends to be poorer yet understudied. According to the America's Health Rankings, Tennessee cancer death rates have increased $7 \%$ since 1990, and the state currently ranks 44th and 45th in health outcomes and cancer deaths nationally [36].

Breast cancer disparities are particularly high in Tennessee. Tennessee women experience the 12th highest breast cancer mortality-to-incidence ratio compared to other women in all 50 U.S. states [37]. Overall, breast cancer mortality rates are about $40 \%$ higher in Black women compared with White women [38]. Black women in Tennessee rank first in breast cancer mortality nationally [39]. Few studies have assessed cancer incidence patterns by Appalachian designation or residence in the state.

According to Penchansky et al. [40] and Gold [41], there are two dimensions of healthcare access. The first is economic access which refers to affordability and the second is geographic access which includes reasonable distance travel time to providers. Studies on geographic access also suggest that people usually seek healthcare in places that are nearer to them than those that are greater distance away [42]. Similarly, Williams et al. [43] also concluded that people may be discouraged from seeking early medical care if they are to travel distances. While the place of residence/geography alone cannot determine the risk of cancer, it can impact prevention, diagnosis, and treatment opportunities. Tennessee, in particular, has been understudied in the area of cancer disparities, notwithstanding, its unique characteristics/classification as both Appalachia and non-Appalachian state. Considering this, the goal of this analysis was to explore the geographic disparities patterns in breast cancer incidence in Tennessee by Appalachian and non-Appalachian county of residence/designation.

\section{Methods}

\section{Data source and study population}

The study population includes all female Tennessee residents aged $\geq 18$ years diagnosed with histologically confirmed breast cancer as the primary site of diagnosis as coded by the International Classification of Diseases for Oncology, Third Edition (ICD-O-3), and reported to the Tennessee Cancer Registry (TCR) from January 1, 2005 to December 31, $2015(N=62,481)$. Detail information about TCR and the dataset is described at https://www. tn.gov/health/health-program-areas/tcr/tennessee-cancer-registry-data.html and in previously publication [44]. Of the total 62,481 cases, Men $[n=643(1.3 \%)]$, other races $[\mathrm{n}=838(1.3 \%)]$, unknown and other missing $[n=1713(2.7 \%)]$ information were excluded. Analysis was performed on a total of 59,278 breast cancer cases. Data used for this analysis is restricted but available by request to the Tennessee Department of HealthTCR at https://www.tn.gov/education/data/data-downl oads/request-data.html. Analytical files are available by reasonable request to and approval by the Tennessee Department of Health (TDH). The Tennessee Department of Health Institutional Review Board approved the research protocol.

\section{Individual-level characteristics}

Individual-level sociodemographic variables obtained from TCR included derived stage at diagnosis, age, race, marital status, type of health insurance, and county of residence. The main outcome stage at diagnosis was categorized as 'early' (defined as in situ and localized stages) and 'late' (defined as regional and distant stages). Age was dichotomized as $<50 ; 50-59 ; 60-69$ and $\geq 70$ years. According to the U.S. Census Burau, the racial/ethnic composition of Tennessee is $73.0 \%$ White (Non-Hispanic); $16.7 \%$ Black or African American; 0.3\% American Indian and Alaska Native; $1.4 \%$ Asians; 0.1\% Native Hawaiian and Other Pacific Islander; some other race $2.2 \%$; two or more races $1.7 \%$; and $4.6 \%$ Hispanic or Latino [45]. However, due to the small number of cancer cases reported among the other racial groups (American Indian and Alaska Native; Asians; Native Hawaiian \& Other Pacific Islander; \& Hispanic/Latino), the TDH/ TCR did not allow us to use these "other racial/ethnic" groups in the analysis to ensure privacy and confidentiality of the patients. As a result, race was limited to Black and White. Marital status was categorized as single/never married; married/common-law; divorced/separated; and widow.

Unlike other developed/industrialized countries, the U.S. does not have a universal healthcare coverage, rather the U.S. operates a hybrid system (i.e. publicly funded and private health insurance). Public health insurance 
is a program run by federal, state, or local governments in which people have some or all their healthcare costs paid for by the government (e.g. Medicare, Medicaid, TRICARE etc.). Medicare is a federally administered program that provides health insurance for senior citizens $($ age $65+)$ and certain disabled people. This health insurance covers medical expenses such as doctor's visits, hospital stays, drugs, and other treatment. Medicaid covers some low-income individuals, their families or those with disabilities who are below the national poverty line. Private insurance, the dominant form of coverage, is primarily employer-sponsored and/or paid by the individual. Private health insurance is provided by employers which requires the insured person to pay a monthly premium in addition to a co-payment when you receive healthcare. Examples include fee-for-service, Health Maintenance Organizations (HMOs) etc. Subsequently, type of health insurance coverage was categorized as public (Indian Health Service, Medicaid, Medicare, and Veterans'
Affairs including TRICARE,); private (fee for service, Health Maintenance Organization [HMO], Managed Care, and Preferred Provider Organization [PPO]); and self-pay or uninsured. Place/county of residence was categorized into two groups on whether the patient resided in an Appalachian or non-Appalachian county.

\section{Statistical and spatial analysis}

We first obtained the distribution of continuous age variable by mean, standard deviation, and range (lower and upper age at diagnosis). Next, the characteristics of patients categorial variables were summarized using frequencies and percentages. We also assessed the association between the categorial variables using chi-square tests. Fisher's exact tests were used to confirm chi-square tests and p-values reported (Table 1). The data was then imported into Environmental Systems Research Institute (ESRI) ArcGIS 10.7 to generate a map of all breast cancer incidence cases in Tennessee. ArcGIS is a geospatial

Table 1 Characteristics of breast cancer patients $(N=59,287)$

\begin{tabular}{|c|c|c|c|}
\hline & Mean & SD & \\
\hline \multirow[t]{2}{*}{ Age at diagnosis (Range: 18,105 ) } & 61.5 & 13.7 & \\
\hline & Number & Percent (\%) & $P$-value \\
\hline \multicolumn{4}{|l|}{ Stage at diagnosis } \\
\hline Early (in situ and localized) & 41,267 & 69.6 & \\
\hline Late (regional and distant) & 18,011 & 30.4 & \\
\hline Race & & & 0.9797 \\
\hline White (ref) & 50,716 & 85.5 & \\
\hline Black & 8571 & 14.5 & \\
\hline Age at diagnosis & & & 0.9912 \\
\hline$<50$ & 11,334 & 19.1 & \\
\hline $50-59$ & 14,393 & 24.3 & \\
\hline $60-69$ & 14,975 & 25.3 & \\
\hline$\geq 70$ (ref) & 18,585 & 31.3 & \\
\hline Marital status* & & & $<0.0001$ \\
\hline Single/never married (ref) & 6859 & 13.0 & \\
\hline Married/common law & 30,947 & 58.6 & \\
\hline Divorced/separated & 6216 & 11.8 & \\
\hline Widowed & 8814 & 16.6 & \\
\hline Type of health insurance* & & & $<0.0001$ \\
\hline Self-pay/uninsured (ref) & 690 & 1.35 & \\
\hline Public & 27,630 & 54.2 & \\
\hline Private & 22,648 & 44.4 & \\
\hline County of residence & & & 0.8076 \\
\hline Non-Appalachian (ref) & 31,096 & 52.4 & \\
\hline Appalachian & 28,191 & 47.6 & \\
\hline
\end{tabular}

Bold implies marital status and type of insurance coverage are statistically significantly associated among women diagnosed with breast cancer in Tennessee Public Insurance (Indian Health Service, Medicaid, Medicare, and Veterans' Affairs including TRICARE)

Private Insurance (fee for service, HMO, Managed Care, and PPO)

$\mathrm{R}$, Range; SD, Standard deviation

* Total values not equal to full sample due to missing data 
software to view create, manage, share, and analyse spatial/geographic data. ESRI develops ArcGIS for mapping on desktop, mobile, and web. Using the 2010 Tennessee population census, age-adjusted incidence rates were calculated by county. Step by step guidance of age adjusted calculation is shown at https://seer.cancer.gov/seerstat/ tutorials/aarates/step1.html. The calculated rates represent the cumulative rate for the 11-year period. To visually compare rates in non-Appalachian $(n=43)$ and Appalachian $(n=52)$ counties, a boarder was generated around the Appalachian counties. Reference points were added to visualize the five major cities in the state. Statistical analyses were conducted using SAS statistical software, version 9.4 (Cary, NC), while spatial analyses were conducted using ESRI ArcGIS 10.7.

\section{Results}

\section{Patients sociodemographic descriptive}

Table 1 provides summary descriptive statistics on breast cancer cases diagnosed from 2005 to 2015 for all female (White and Black/African American) residents aged 18 years and older in Tennessee. Patient ages range from 18 to 105 years. The mean age at diagnosis for the whole sample was 61.5 years old with a standard deviation (SD) of 13.7. Most women in the study (85.5\%; $\mathrm{n}=50,716)$ were White, married (58.6\%; $n=30,947)$, aged $\geq 70$ $(31.3 \% ; 18,585)$, and diagnosed with an early stage of breast cancer $(69.6 \% ; n=41,267)$. More than half of the women had public health insurance $(54.2 \% ; \mathrm{n}=27,630)$, followed by private health insurance coverage (44.4\%; $\mathrm{n}=22,648)$. Over half of the women $(\mathrm{n}=31,096)$ resided in non-Appalachian, whereas $47.6 \%(n=28,191)$ were in Appalachia. We observed a significant association among breast cancer patients with respect to marital status and type of health insurance coverage $(p=<0.0001)$. See Table 1.

\section{Spatial distribution by county of residence}

The spatial analysis map depicts the overall breast cancer incidence age-adjusted rates across the 95 counties from 2005 to 2015 (See Fig. 2). Incidence rates are displayed along a color gradient, meaning the darkest colors represent the highest rates, while the lightest the lowest. Although reported breast cancer incidences were slightly greater in non-Appalachian counties, the ageadjusted results show higher rates in the Appalachian counties compared to non-Appalachian counties. Also, we observe a clustering of mid-to-high rates around Nashville (central region), Knoxville, and Johnson City (northeast region). The highest age-adjusted rates of 997.49-1164.59/100,000 were recorded in 9 counties with 6 in Appalachia (Anderson, Blount, Knox, Rhea, Roane, and Van Buren), and 3 in non-Appalachia (Fayette, Marshall, and Williamson). To establish spatial autocorrelation, we ran a test for Moran's index and found a correlation between the clustering of similar high and

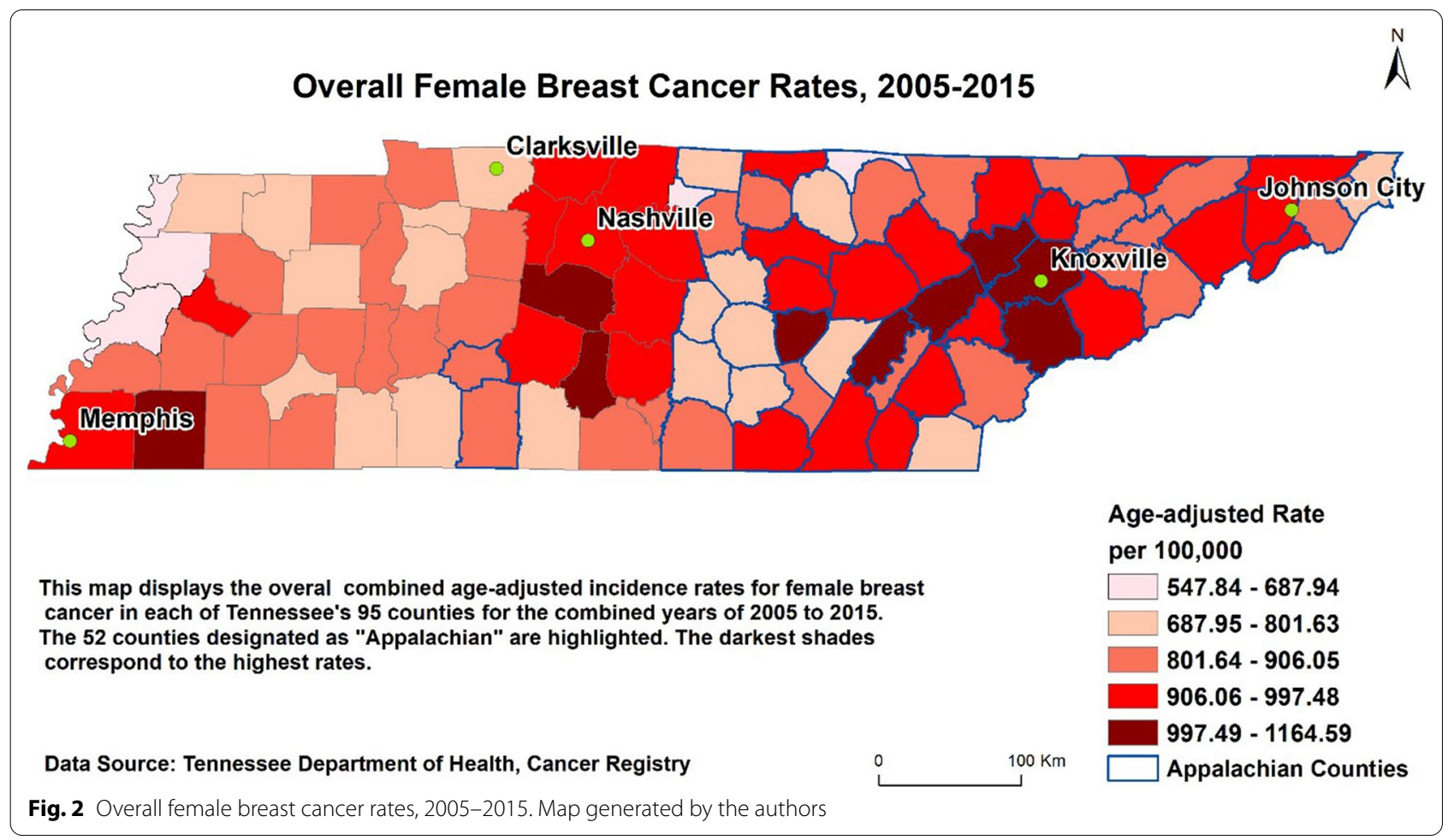


low rate counties $(\mathrm{MI}=0.203, p=0.012)$. Further, the map also indicated that regardless of Appalachian designation, clustering of the highest rates was reported near major cities, i.e. Johnson City and Knoxville (Appalachian), Memphis, and Nashville (non-Appalachian) (see Fig. 2).

Figure 2 shows combined age-adjusted incidence rates for female breast cancer in each of Tennessee's 95 counties from 2005-2015

\section{Discussion}

The Appalachian region of the U.S. represents $8 \%$ (i.e. $>25$ million) of the total population [3]. The region is a National Cancer Institute-designated priority area as it represents one of the most economically disadvantaged, medically underserved regions, and experiences elevated breast cancer risk and other disproportionate cancer burdens $[10,46]$. Complex and interrelated set of individual, interpersonal, community, and societal factors are known to contribute to health inequities and disparities in breast cancer incidence, diagnosis and mortality in the Appalachian region [47-49]. The goal of this snapshot was to enhance our understanding of breast cancer incidence distribution by Appalachian county of residence and determine if geographic disparities exist in Tennessee.

Age is one of the important risk factors for breast cancer, and most breast cancer cases are diagnosed in women $\geq 50$ years old. However, about $11 \%$ of breast cancer cases occur in women below age $<45$ in the U.S. when the disease is more aggressive leading to poorer prognosis and survival compared to older women [50]. Previously, Partridge et al. [51] examined the effect of age, and delay in breast cancer stage at diagnosis using the National Comprehensive Cancer Network (NCCN) Breast Cancer Outcomes Database Project. They found that women aged $\leq 40$ had a $50 \%$ (OR 1.52; 95\% CI 1.391.67; $\mathrm{p}<0.0001$ ) higher likelihood of $>60$ days delay in breast cancer diagnosis than older women [51]. Another study from Sweden evaluated the relationship between age and breast cancer and found that age was positively associated with high breast cancer-specific mortality especially among women $<40$ years old [52]. In the present study, between 2005 and 2015 more breast cancer cases were diagnosed in women $\geq 50$ years compared to younger women under age 50 . The highest number of cases was reported among women $\geq 70$ years $(31.3 \%$ ), followed by women aged 50-59 (24.3\%), and 60-69 (25.35) year, however, the association was not significant $(p=0.9912)$ among women diagnosed with breast cancer in Tennessee. There was also no significant difference between White women and Black women diagnosed with breast cancer $(p=0.9797)$ in Tennessee.
Studies have reported a lower breast cancer risk for married women than single/unmarried women [5356]. Factors attributed to this include financial stability, engaging in physical activity, healthy behavioral lifestyles like better nutrition, family support, adherence to recommended screening and treatment [53-56]. In contrast, Barry and Breen reported that marital status and being Black both contribute to late-stage diagnosis in Atlanta, GA [57]. Specifically, in the city of Detroit, being unmarried, being Black, and residing in an area of extreme poverty were all significant [57]. Health insurance is also identified as a predictor of access and use of healthcare services [54, 58-60]. In our study, we found marital status and type of health insurance coverage played a significant role in the diagnosis of breast cancer.

Place of residence, neighborhood socioeconomic characteristics, and built environments are recognized as important contexts through which our health is shaped. Previous studies [2, 26, 61-68], reported extraordinarily high cancer rates and health disparities in Appalachia and rural America. A recent Centers for Disease Control and Prevention's (CDC) Morbidity and Mortality Weekly Report noted that rural America experienced an increase in cancer deaths from 2006 to 2015 despite a lower incidence (2004-2013) compared to urban America [68]. Using the National Program of Cancer Registries linked with Medicare claims from Pennsylvania, Ohio and Kentucky, Anderson et al. [64], analyzed breast cancer screening, geographic deprivation, and late stage diagnosis. The authors revealed that counties with the highest economic deprivation reported the least screening, and the highest rates of late-stage diagnosis (21.05 vs 15.10 vs 17.17). Kentucky the state with the most deprived Appalachian counties, reported the strongest association compared to Pennsylvania, and Ohio counties [64]. Similarly, a systematic review that examined the relationship between place of residence and late breast cancer incidence found that almost 50\% (17/36) of the eligible studies indicated breast cancer patients residing in geographically remote/rural areas had more late-stage diagnosis than urban women [2].

While our reported proportion of breast cancer incidence in the non-Appalachian counties $(31,096 ; 52.4 \%)$ was slightly higher than Appalachian counties $(28,191$; $47.6 \%)$, the difference was not significant $(p=0.8076)$. On the other hand, Bennett et al. [69] analyzed the effect of urban versus rural residence on stage at diagnosis and survival among women with breast cancer in Aotearoa, New Zealand. Results showed that living in an urban or rural area did not have any statistically significant effect on breast cancer stage at diagnosis or survival [69]. However, consistent with previous findings from the U.S., and across the world [70,71], our spatial analysis confirms 
geographical disparities exist between Appalachian and non-Appalachian counties in the overall age-adjusted breast cancer rates mainly around central and eastern Tennessee. Tin et al. [70] examined ethnic disparities in breast cancer survival with Māori (indigenous people) and Pacific women (immigrants or descended from immigrants from Pacific Islands women). They reported that over half of Maori and Pacific women lived in the most or predominantly deprived neighborhoods and were twice as likely to die from breast cancer when compared to non-Maori non-Pacific women [70]. Likewise, Valery et al. [71] investigated the effect of stage at diagnosis, treatment, and comorbidities on survival between non-Indigenous and Indigenous cancer patients. Results showed that compared to non-Indigenous patients, Indigenous patients in Queensland, New Zealand, were 1.5 times more likely (95\% CI $1.3-1.7)$ to die from the disease [71].

Rural/urban disparities in breast cancer incidence/ diagnosis, treatment, mortality, and survival have been reported among minority populations and other underprivileged groups around the world. Although the underlying causes for the observable breast cancer outcomes are complex and not well understood some authors have attributed it to geography, economic or political condition, limited resources and inequity in healthcare access and quality [72, 73]. For instance, many Appalachian areas are isolated from larger cities limiting ease and equitable access to required medical resources as already noted. However, Tennessee is a unique state as major metropolitan cities Johnson City, Kingsport, and Knoxville fall within the Appalachian region, providing rural/ urban diversity not seen in many other Appalachia states like Kentucky and West Virginia. As a result, expanding healthcare resources in these urban Appalachian metropolitan cities may benefit the state's Appalachian communities and enhance breast cancer screening as well as early detection, a potential factor in the increased incidence rates observed in these areas.

Our findings serve to provide important public health implications not just for the Appalachian/rural regions of the United States, but other parts of the world that share similar socioeconomic and geographic characteristics. As previously mentioned, place of residence, neighborhood socioeconomic characteristics, and built environment are major factors in shaping health. Geographic differences in breast cancer incidence found in this study can be used by local public health officials to focus prevention efforts on counties that are in need and would benefit the most from strategies aimed at preventing cancer from occurring and reduce disparities. When examining other resource-limited areas around the world, the implementation of methods used in this study can draw attention to areas of need. Anderson et al. [74] previously described the barriers to breast cancer care in limitedresource settings as being a lack or recognition of cancer as a public health priority. Additional issues such as trained healthcare personnel shortages and migration, public and healthcare provider educational deficits, and social barriers may also impede patient entry into early detection and cancer treatment programs [74]. To bridge the disparities gap, identifying the scope of the problem in underserved or limited-resource areas (i.e. the Appalachian region and similar places) is an important step in controlling and reducing barriers to breast cancer diagnosis and fatality around the world.

\section{Strengths and limitations}

This study adds to the existing literature by using the geographic information system to explore breast cancer incidence patterns among women living in Appalachian Tennessee and non-Appalachian Tennessee counties. We found a higher overall age-adjusted incidence rate among women in Appalachian counties compared to non-Appalachian counties. This is an important finding considering the unique geographical and social characteristics of Tennessee, a state not covered in the Surveillance, Epidemiology, and End Results program (SEER) [75]. Further, as determinants of health such as environmental, socio-cultural, and physical environment differ greatly from place to place, so do people's healthcare needs. The strength of the geospatial analysis is shown through the insignificant result of breast cancer and county of residence in t-tests. However, significantly higher cluster rates were observed among Appalachian counties using spatial analysis. We would not have been able to determine this relationship without the techniques we used. It is important to point out that rural urban differences in breast cancer incidence are not the same everywhere and may differ within countries, regions, states, or counties and should not be generalized. Therefore, the use of Geographic Information System (GIS) tools is useful to understand public health issues in any area/geography, as well as provide useful information in decision making. In particular GIS can serve as an essential tool for researchers, social scientists, health educators to understand their population. It is also a powerful tool for health planning, monitoring and the evaluation of the effectiveness of health programs aimed at reducing premature disability and death due to breast cancer, in Tennessee and in similar settings around the world.

Nonetheless, our study is not without limitation. First, we were limited by the retrospective administrative variables available to us. For instance, cancer registries do not collect socioeconomic status data (e.g., income, education, etc.), behavior/lifestyle factors (e.g. smoking, 
nutrition/diet, etc.), and quality of treatment received by patients. Sociodemographic variables such as age, race, insurance status, and marital status made available to use were only collected at the time of diagnosis and may not be up to date. Second, the study focused on a single state in the United States, Tennessee, which limits its generalizability to another state, geographic location, region, or country different from Tennessee. Despite, these limitations, the findings are essential since they provide a better understanding of place of residence and breast cancer disparities for Tennessean women and provide methods for establishing a baseline of geographic variation in cancer incidence.

\section{Conclusions}

This study used Geographic Information System to investigate breast cancer incidence patterns by Appalachian county designation in Tennessee. We observed spatial disparities in the Appalachian county of residence, as well as significant relationship in marital status, type of health insurance, and breast cancer through the univariate analysis but did not show an association by age at diagnosis, race, and county of residence. As a next step, future studies will continue to utilize GIS methods to examine the relationship between the location of healthcare facilities and distance traveled (transportation) and the potential effect on cancer treatment, mortality, and quality of life. We will also link the cancer incidence-to-mortality as well as medical record data to assess survival outcomes by sociodemographic characteristics, socioeconomic status, and other lifestyle behaviors. Further, additional research should focus on other neighborhood-level factors that may impact cancer health outcomes, outside of Appalachian designation, in addition to an examination of screening trends on incidence and mortality.

\section{Abbreviations \\ CDC: Centers for Disease Control and Prevention; ESRI: Environmental Systems Research Institute; GIS: Geographic Information System; HMO: Health Mainte- nance Organization; ICD-O-3: International Classification of Diseases for Oncol- ogy, Third Edition; NCCN: National Comprehensive Cancer Network; PPO: Preferred Provider Organization; SD: Standard deviation; SEER: Surveillance, Epidemiology, and End Results program; TCR: Tennessee Cancer Registry; TDH: Tennessee Department of Health; VA: Veterans' Affairs.}

\section{Acknowledgements}

Tennessee Department of Health data used in this study was obtained from the Office of Cancer Surveillance, Tennessee Department of Health (TDH). Use of these data does not imply TDH agrees or disagrees with any presentations, analyses, interpretations, or conclusions herein.

\section{Authors' contributions}

FW conceived, designed, and acquired the data. BS and LM cleaned, managed, analyzed, and interpretation of results under FW supervision. BS drafted the manuscript, supervised by FW. LM, XL and MW substantially modified the submitted version of the manuscript. All authors read and approved the final version of the manuscript.

\section{Funding}

Open Access funding provided by the National Institutes of Health (NIH). Ms. Salmeron, Drs. Liu, and Williams' efforts were supported by the Division of Intramural Research, National Institute on Minority Health and Health Disparities, National Institutes of Health. The content is solely the responsibility of the authors and does not necessarily reflect the views of the National Institutes of Health.

\section{Availability of data and materials}

Data used for this analysis are restricted but available by request to the Tennessee Department of Health, Tennessee Cancer Registry (https://www.tn. gov/education/data/data-downloads/request-data.html). All analytical files are available by reasonable request and per Tennessee Department of Health approval.

\section{Declarations}

Ethics approval and consent to participate

The research protocol and access to the data for this study went to full review by the Tennessee Department of Health Institutional Review Board and was approved on 01 February 2018 (TDH-IRB 1057486). The National Institutes of Health - Intramural Research Program IRB - Human Research Protections Program - Office of Human Subjects Research Protections determined that our protocol did not involve human subjects and was excluded from IRB review (18-NIMHD-00722).

\section{Consent for publication}

Not applicable.

\section{Competing interests}

The authors declare no conflict of interest.

\section{Author details}

${ }^{1}$ Division of Intramural Research, National Institute on Minority Health and Health Disparities, Bethesda, MD, USA. ${ }^{2}$ Department of Mathematics and Statistics, University of South Florida, Tampa, FL, USA. ${ }^{3}$ Tennessee Department of Health, Office of Cancer Surveillance, Nashville, TN, USA.

Received: 16 September 2020 Accepted: 18 March 2021

Published online: 03 May 2021

\section{References}

1. Hunt BR, Whitman S, Hurlbert MS. Increasing Black: White disparities in breast cancer mortality in the 50 largest cities in the United States. Cancer Epidemiol. 2014;38(2):118-23.

2. Williams F, Jeanetta $S$, James AS. Geographical location and stage of breast cancer diagnosis: a systematic review of the literature. J Health Care Poor Underserved. 2016;27(3):1357-83.

3. The Appalachian Region https://www.arc.gov/appalachian_region/ TheAppalachianRegion.asp. Accessed 17 Dec 2020.

4. Marshall J, Thomas L, Lane N, Holmes G, Arcury T, Randolph R, Silberman P, Holding W, Villamil Lea: Health Disparities in Appalachia August 2017: Creating a culture of health in Appalachia: disparities and bright spots https://www.arc.gov/research/researchreportdetails.asp? REPORT_ID=138. Accessed 16 July 2019.

5. Behringer B, Friedell GH, Dorgan KA, Hutson SP, Naney C, Phillips A, Cantrell E. Understanding the challenges of reducing cancer in Appalachia: addressing a place-based health disparity population. Calif J Health Promot. 2007:5:40-9.

6. Woolley SM, Meacham SL, Balmert LC, Talbott EO, Buchanich JM. Comparison of mortality disparities in central Appalachian coal-and non-coal-mining counties. J Occup Envrion Med. 2015;57(6):687-94.

7. Yao N, Alcalá HE, Anderson R, Balkrishnan R. Cancer disparities in rural appalachia: incidence, early detection, and survivorship. J Rural Health. 2016;33(4):375-81.

8. Siegel RL, Miller KD, Jemal A. Cancer statistics, 2020. CA Cancer J Clin. 2020;70(1):7-30. 
9. Lengerich EJ, Kluhsman BC, Bencivenga M, Anderson M. The northern appalachia cancer network: changing cancer research, changing people's lives. J High Educ Outreach Engagem. 2010;14(3):85-95.

10. Lengerich EJ, Tucker TC, Powell RK, Colsher P, Lehman E, Ward AJ, Siedlecki JC, Wyatt SW. Cancer incidence in Kentucky, Pennsylvania, and West Virginia: disparities in Appalachia. J Rural Health. 2005;21 (1):39-47.

11. Network ACC: Addressing the cancer burden in Appalachian communities 2010.

12. Yao N, Matthews SA, Hillemeier MM, Anderson RT. Radiation therapy resources and guideline-concordant radiotherapy for early-stage breast cancer patients in an underserved region. Health Serv Res. 2013;48(4):1433-49.

13. Barker L, Crespo R, Gerzoff RB, Denham S, Shrewsberry M, CorneliusAverhart D. Residence in a distressed county in Appalachia as a risk factor for diabetes, behavioral risk factor surveillance system, 2006-2007. Prev Chronic Dis. 2010;7(5):A104.

14. Central Appalachia. Central Appalachia Region. http://www.ruralhome. org/storage/documents/appalov.pdf. Accessed 21 Feb 2021.

15. Borak J, Salipante-Zaidel C, Slade MD, Fields CA. Mortality disparities in appalachia: reassessment of major risk factors. J Occup Environ Med. 2012;54(2):146-56.

16. Appalachian Regional Commission (ARC). Poverty Rates in Appalachia, 2010-2014 https://www.arc.gov/research/MapsofAppalachia.asp?MAP_ $\mid \mathrm{D}=121$. Accessed 17 Dec 2020

17. Appalachian Regional Commission (ARC). Subregions in Appalachia https://www.arc.gov/research/MapsofAppalachia.asp?MAP_ID=31. Accessed 5 Feb 2021.

18. Brown KC, Fitzhugh EC, Neutens JJ, Klein DA. Screening mammography utilization in Tennessee women: the association with residence. J Rural Health. 2009;25(2):167-73.

19. Halverson J, Ma L, Harner E. An analysis of disparities in health status and access to health care in the Appalachian region: executive summary. Washington: Appalachian Regional Commission; 2004.

20. Knight JR, Huang B, Tucker T. Breast cancer in Kentucky: progress and possibilities. Dr Alvarado Goes to Frankfort. 2015. p. 93.

21. Lyttle NL, Stadelman K. Assessing awareness and knowledge of breast and cervical cancer among Appalachian women. Prev Chronic Dis. 2006;3(4):A125.

22. Lopez ED, Khoury AJ, Dailey AB, Hall AG, Chisholm LR. Screening mammography: a cross-sectional study to compare characteristics of women aged 40 and older from the deep South who are current, overdue, and never screeners. Womens Health Issues. 2009;19(6):434-45.

23. Schoenberg NE, Studts CR, Hatcher-Keller J, Buelt E, Adams E. Patterns and determinants of breast and cervical cancer non-screening among Appalachian women. Women Health. 2013;53(6):552-71.

24. Hatcher J, Dignan MB, Schoenberg N. How do rural health care providers and patients view barriers to colorectal cancer screening? Insights from Appalachian Kentucky. Nurs Clin Noth Am. 2011;46(2):181-92.

25. Schoenberg NE, Bardach SH, Manchikanti KN, Goodenow AC. Appalachian residents' experiences with and management of multiple morbidity. Qual Health Res. 2011;21(5):601-11.

26. Yao N, Lengerich EJ, Hillemeier MM. Breast cancer mortality in Appalachia: reversing patterns of disparity over time. J Health Care Poor Underserved. 2012;23(2):715-25.

27. McMillan SJ, Haley E, Zollman-Huggler P, Johnson Avery E, Winchenbach MG, Bell JL. Breast health education for working women in Appalachia: insights from focus group research. Cancer Control. 2007;14(3):265-76.

28. Hall AG, Khoury AJ, Lopez ED, Lisovicz N, Avis-Williams A, Mitra A. Breast cancer fatalism: the role of women's perceptions of the health care system. J Health Care Poor Underserved. 2008;19(4):1321-35.

29. Hutson SP, Dorgan KA, Phillips AN, Behringer B. The mountains hold things in the use of community research review work groups to address cancer disparities in Appalachia. Oncol Nurs Forum. 2007;34(6):1133-9.

30. LeMasters T, Madhavan S, Atkins E, Vyas A, Remick S, Vona-Davis L. "Don't know" and accuracy of breast cancer risk perceptions among Appalachian women attending a mobile mammography program: implications for educational interventions and patient empowerment. J Cancer Educ. 2014;29(4):669-79.

31. Schoenberg NE, Kruger TM, Bardach S, Howell BM. Appalachian women's perspectives on breast and cervical cancer screening. Rural Remote Health. 2013;13(3):2452.
32. VanDyke SD, Shell MD. Health beliefs and breast cancer screening in rural Appalachia: an evaluation of the health belief model. J Rural Health. 2016.

33. Blackley D, Behringer B, Zheng S. Cancer mortality rates in Appalachia: descriptive epidemiology and an approach to explaining differences in outcomes. J Commun Health. 2012;37(4):804-13.

34. lqbal J, Ginsburg O, Rochon PA, Sun P, Narod SA. Dlfferences in breast cancer stage at diagnosis and cancer-specific survival by race and ethnicity in the united states. JAMA. 2015;313(2):165-73.

35. Reeder-Hayes KE, Wheeler SB, Mayer DK. Health disparities across the breast cancer continuum. Semin Oncol Nurs. 2015;31(2):170-7.

36. United Health Foundation. America's Health Rankings. https://www.ameri cashealthrankings.org/explore/annual/measure/CancerDeaths/state/TN. Accessed 17 July 2019.

37. Tennessee Department of Health. Breast cancer learning community. https://www.astho.org/Prevention/Chronic-Disease/Breast-Cancer-Dispa rities/Learning-Community/Tennessee-One-Pager-Summary/. Accessed 17 Apr 2020.

38. Richardson LC, Henley SJ, Miller JW, Massetti G, Thomas CC. Patterns and trends in age-specific black-white differences in breast cancer incidence and mortality-United States, 1999-2014. MMWR Morb Mortal Wkly Rep. 2016;65(40):1093-8.

39. Hunt BR, Silva A, Lock D, Hurlbert M. Predictors of breast cancer mortality among white and black women in large United States cities: an ecologic study. Cancer Causes Control. 2019;30(2):149-64.

40. Penchansky R, Thomas JW. The concept of access: definition and relationship to consumer satisfaction. Med Care. 1981;19(2):127-40.

41. Gold M. Beyond coverage and supply: measuring access to healthcare in today's market. Health Serv Res. 1998;33(3 Pt 2):625.

42. Brustrom JE, Hunter DC. Going the distance: how far will women travel to undergo free mammography? Military Med. 2001;166(4):347-9.

43. Williams AP, Schwartz WB, Newhouse JP, Bennett BW. How many miles to the doctor? N Engl J Med. 1983;309(16):958-63.

44. Montiel Ishino FA, Rowan C, Das R, Thapa J, Cobran E, Whiteside $\mathrm{M}$, Williams F. Identifying risk profiles of malignant prostate cancer surgical delay using a person-centered approach to understand prostate cancer disparities: the constellation of health determinants using latent class analysis on cancer registry data. Am J Mens Health. 2020;14(6):1557988320984282.

45. United States Census Bureau. 2010 Census: Tennessee profile. https:// www2.census.gov/geo/maps/dc10_thematic/2010_Profile/2010_Profile_ Map_Tennessee.pdf. Accessed 30 Jan 2021.

46. Braun KL, Stewart S, Baquet C, Berry-Bobovski L, Blumenthal D, Brandt HM, Buchwald DS, Campbell JE, Coe K, Cooper LC, et al. The National Cancer Institute's community networks program initiative to reduce cancer health disparities: outcomes and lessons learned. Prog Commun Health Partnersh. 2015;9:21-32.

47. Lamb EP, Pritchard FE, Nouer SS, Tolley EA, Boyd BS, Davidson JT, Munene $\mathrm{G}$, Fleming MD. Understanding disparities in breast cancer care in Memphis, Tennessee. Am Surg. 2018;84(5):620-7.

48. Patel K, Kanu M, Liu J, Bond B, Brown E, Williams E, Theriot R, Bailey S, Sanderson M, Hargreaves M. Factors influencing breast cancer screening in low-income African Americans in Tennessee. J Commun Health. 2014:39:943-50.

49. Williams F, Colditz GA, Hovmand P, Gehlert S. Combining communityengaged research with group model building to address racial disparities in breast cancer mortality and treatment. J Health Dispar Res Pract. 2018;11(1):160-78.

50. Centers for Disease Control and Prevention. Breast cancer in young women. https://www.cdc.gov/cancer/breast/young_women/bringyourb rave/index.htm. Accessed 15 Sept 2020.

51. Partridge AH, Hughes ME, Ottesen RA, Wong Y-N, Edge SB, Theriault RL, Blayney DW, Niland JC, Winer EP, Weeks JC, et al. The effect of age on delay in diagnosis and stage of breast cancer. Oncologist. 2012;17(6):775-82.

52. Brandt J, Garne JP, Tengrup I, Manjer J. Age at diagnosis in relation to survival following breast cancer: a cohort study. World J Surg Oncol. 2015;13(1):33.

53. Gomez SL, Hurley S, Canchola AJ, Keegan TH, Cheng I, Murphy JD, Clarke CA, Glaser SL, Martínez ME. Effects of marital status and economic resources on survival after cancer: a population-based study. Cancer. 2016;122(10):1618-25. 
54. Hsu CD, Wang X, Habif DV Jr, Ma CX, Johnson KJ. Breast cancer stage variation and survival in association with insurance status and sociodemographic factors in US women 18 to 64 years old. Cancer. 2017;123(16):3125-31

55. Li M, Han M, Chen Z, Tang Y, Ma J, Zhang Z, Liu Z, Zhang N, Xi C, Liu J, et al. Does marital status correlate with the female breast cancer risk? A systematic review and meta-analysis of observational studies. PLoS ONE. 2020;15(3):e0229899-e0229899.

56. Martínez ME, Unkart JT, Tao L, Kroenke CH, Schwab R, Komenaka I, Gomez SL. Prognostic significance of marital status in breast cancer survival: a population-based study. PLoS ONE. 2017;12(5):e0175515-e0175515.

57. Barry J, Breen N. The importance of place of residence in predicting late-stage diagnosis of breast or cervical cancer. Health Place. 2005;11(1):15-29.

58. Dickman SL, Himmelstein DU, Woolhandler S. Inequality and the healthcare system in the USA. Lancet. 2017;389(10077):1431-41.

59. Pan HY, Walker GV, Grant SR, Allen PK, Jiang J, Guadagnolo BA, Smith BD, Koshy M, Rusthoven CG, Mahmood U. Insurance status and racial disparities in cancer-specific mortality in the United States: a population-based analysis. Cancer Epidemiol Biomarkers Prev. 2017;26(6):869-75.

60. Wilson SL, Kratzke C, Hoxmeier J. Predictors of access to healthcare: what matters to rural Appalachians? Glob J Health Sci. 2012;4(6):23-35.

61. Dai D. Black residential segregation, disparities in spatial access to health care facilities, and late-stage breast cancer diagnosis in metropolitan Detroit. Health Place. 2010;16:1038-52.

62. Dwyer-Lindgren L, Bertozzi-Villa A, Stubbs RW, et al. Us county-level trends in mortality rates for major causes of death, 1980-2014. JAMA. 2016;316(22):2385-401.

63. Yao N, Alcalá HE, Anderson R, Balkrishnan R. Cancer disparities in rural Appalachia: incidence, early detection, and survivorship. J Rural Health. 2017;33(4):375-81.

64. Anderson RT, Yang TC, Matthews SA, Camacho F, Kern T, Mackley HB, Kimmick G, Louis C, Lengerich E, Yao N. Breast cancer screening, area deprivation, and later-stage breast cancer in Appalachia: does geography matter? Health Serv Res. 2014;49(2):546-67.

65. Dasgupta P, Baade PD, Aitken JF, Turrell G. Multilevel determinants of breast cancer survival: association with geographic remoteness and area-level socioeconomic disadvantage. Breast Cancer Res Treat. 2012;132(2):701-10.
66. Williams F, Jeanetta $S$, O'Brien D, Fresen J. Rural-urban difference in female breast cancer diagnosis in Missouri. Rural Remote Health. 2015;15(3063):206.

67. Baade PD, Turrell G, Aitken JF. Geographic remoteness, area-level socioeconomic disadvantage and advanced breast cancer: a cross-sectional, multilevel study. J Epidemiol Commun Health. 2011;65(11):1037-43.

68. Henley SJ, Anderson RN, Thomas CC, Massetti GM, Peaker B, Richardson LC. Invasive cancer incidence, 2004-2013, and deaths, 2006-2015, in nonmetropolitan and metropolitan counties-United States. MMWR Surveill Summ. 2017;66(14):1.

69. Bennett $H$, Marshall R, Campbell I, Lawrenson R. Women with breast cancer in Aotearoa New Zealand: the effect of urban versus rural residence on stage at diagnosis and survival. N Z Med J. 2007;120(1266):U2831.

70. Tin Tin S, Elwood JM, Brown C, Sarfati D, Campbell I, Scott N, Ramsaroop $\mathrm{R}$, Seneviratne S, Harvey V, Lawrenson R. Ethnic disparities in breast cancer survival in New Zealand: which factors contribute? BMC Cancer. 2018;18(1):58.

71. Valery PC, Coory M, Stirling J, Green AC. Cancer diagnosis, treatment, and survival in Indigenous and non-Indigenous Australians: a matched cohort study. Lancet. 2006:367(9525):1842-8.

72. Williams F, Thompson E. Disparities in breast cancer stage at diagnosis: importance of race, poverty, and age. J Health Dispa Res Practi. 2017;10(3):4.

73. McGee SA, Durham DD, Tse C-K, Millikan RC. Determinants of breast cancer treatment delay differ for African American and White women. Cancer Epidemiol Biomark Prev. 2013;22(7):1227.

74. Anderson BO, Yip C-H, Ramsey SD, Bengoa R, Braun S, Fitch M, Groot M, Sancho-Garnier H, Tsu VD, Systems ftGSHC et al: Breast cancer in limitedresource countries: health care systems and public policy. Breast J. 2006, 12(s1): S54-S69.

75. National Cancer Institute. Surveilliance, epiedmiology, and end results program (SEER): About the SEER registries https://seer.cancer.gov/regis tries/. Accessed 30 Jan 2021.

\section{Publisher's Note}

Springer Nature remains neutral with regard to jurisdictional claims in published maps and institutional affiliations.
Ready to submit your research? Choose BMC and benefit from:

- fast, convenient online submission

- thorough peer review by experienced researchers in your field

- rapid publication on acceptance

- support for research data, including large and complex data types

- gold Open Access which fosters wider collaboration and increased citations

- maximum visibility for your research: over $100 \mathrm{M}$ website views per year

At BMC, research is always in progress.

Learn more biomedcentral.com/submissions 